\title{
Endometrial cancer prognosis correlates with the expression of L1CAM and miR34a biomarkers
}

\author{
Giacomo Corrado ${ }^{1,5^{*}}$, Valentina Laquintana ${ }^{2 \dagger}$, Rossella Loria ${ }^{2}$, Mariantonia Carosi ${ }^{3}$, Laura de Salvo ${ }^{3}$, \\ Isabella Sperduti ${ }^{4}$, Ashanti Zampa', Lucia Cicchillitti², Giulia Piaggio², Giuseppe Cutillo', Rita Falcioni2 ${ }^{2+}$ \\ and Enrico Vizza ${ }^{1+}$
}

\begin{abstract}
Background: Patients with endometrial cancer (EC) and presumably with good prognosis may develop a recurrence indicating that the classification of this tumor is still not definitive and that new markers are needed to identify a subgroup at risk of relapse. The cell adhesion molecule L1CAM is highly expressed in several human carcinomas and has recently been described as a new marker for endometrial and ovarian carcinomas. The aim of this study was to determine the relevance of L1CAM in recurrent EC.

Methods: In this work we have analyzed, by immunohistochemical and RT-qPCR analysis, the expression of L1CAM in a cohort of 113 endometrial cancers at different stages, which 50\% have relapsed. As a predictor of good outcome, the tumors were also analyzed for the expression of miR-34a, a post-transcriptional regulator of L1CAM.

Results: Among metastatic EC, the highest levels (60\%) and the median level (24\%) of L1CAM in tumors correlate with the progression, suggesting that the expression of this molecule is linked to the tumor component most involved in metastatic processes. We also found an inverse correlation between miR-34a and L1CAM protein expression, suggesting that miR-34a is a positive prognostic marker of EC.
\end{abstract}

Conclusions: Our results demonstrate the expression of L1CAM and miR-34a in EC as prognostic factors that identify subgroup of patients at high risk of recurrence suggesting for them more aggressive schedules of treatment.

Keywords: L1CAM, Endometrial cancer, Prognostic biomarker, Personalised approach, Innovative biotechnology

\section{Background}

Endometrial cancer (EC) is the most common gynecologic malignancy in industrialized countries [1]. From the pathogenic point of view, EC falls into two different types, so-called types I and II, and this classification subsequently has shown systematic differences in molecular features, and consequently, in prognosis and treatment

\footnotetext{
* Correspondence: giacomo.corrado@alice.it

${ }^{\dagger}$ Giacomo Corrado, Valentina Laquintana, Rita Falcioni and Enrico Vizza contributed equally to this work.

'Department of Experimental Clinical Oncology, Gynecologic Oncology Unit, IRCCS - Regina Elena National Cancer Institute, Via Elio chianesi, 53 -, 00144 Rome, Italy

${ }^{5}$ Present address: Department of Women and Children Health, Gynecologic Oncology Unit, Fondazione Policlinico Universitario A. Gemelli - IRCCS, Università Cattolica del Sacro Cuore, Roma, Italy

Full list of author information is available at the end of the article
}

[2]. Within type I EC, or estrogen-dependent endometrioid, the phosphatidylinositide 3-kinases (PI3K)/protein serine-threonine kinase AKT pathway is the most frequently altered, with inactivation of the phosphatase and tensin homolog tumor (PTEN) suppressor gene, which modulates cell division and enables apoptosis. The most common molecular alterations observed in type $2 \mathrm{EC}$ that consists of the estrogen-independent non-endometrioid carcinoma are p53 and p16 mutations, HER2 overexpression or amplification and loss of E-cadherin [3]. The Cancer Genome Atlas (TCGA) Research Network proposed an innovative classification of EC that divided EC into four categories, with probable influence on treatment recommendations, based on an integrated genomic, transcriptomic and proteomic characterization [4]. Despite these

(c) The Author(s). 2018 Open Access This article is distributed under the terms of the Creative Commons Attribution 4.0 International License (http://creativecommons.org/licenses/by/4.0/), which permits unrestricted use, distribution, and 
important innovations to better-characterized EC, no effective biomarkers currently exist to direct treatment (adjuvant radiation and/or chemotherapy) in EC, or to triage pelvic and para-aortic lymphadenectomy. In the last years, L1-cell adhesion molecule (L1CAM), a transmembrane protein of the immunoglobulin family that has been implicated in promoting tumor cell proliferation, migration, invasion, and metastasis, has been investigated in EC [5]. The immunohistochemical (IHC) detection of L1CAM in endometrial tumor samples seems to be able to discriminate a subset of highly aggressive tumors with high risk of distant recurrences [6] and to assess the risk of pelvic lymph-node involvement [7]. Moreover, it has been previously reported that miR34-a controls the expression of L1CAM inducing its mRNA degradation [8]. Specifically, it was demonstrated that the overexpression of miR-34a in EC cell line abrogates L1CAM expression and, as consequence, inhibits cell migration [8].

We therefore set out to determine the relevance of L1CAM and miR34-a in recurrent EC, which includes both type $<1 \mathrm{~B}$ and $\geq 1 \mathrm{~B}$.

\section{Methods}

\section{Patients cohort}

Retrospective cohorts of formalin-fixed paraffin embedded (FFPE) specimens, derived from 113 patients with EC surgically treated at the Regina Elena National Cancer Institute, were collected from $24 / 12 / 2001$ to $05 / 12 / 2014$. The patients of the two cohorts (57 with recurrent and 56 non-recurrent) were selected from a large database with propensity score matching method. The two subsets were matched for potentially confounders: age, stage, grading and histotype. The study was reviewed and approved by the ethical committee of "Regina Elena" National Cancer Institute, and informed consent was obtained from all patients.

\section{Quantitative RT-PCR}

Total RNA, derived from EC, was isolated by PureLink FFPE kit (Invitrogen), and reverse-transcribed using PrimeScript RT reagent kit (Takara). Quantitative PCR (RT-qPCR) was performed using SYBR Green on an ABI Prism 7500 apparatus (Applied Biosystems, Glasgow, $\mathrm{UK})$ in 2 independent experiments in triplicate. The comparative threshold $(\Delta \mathrm{Ct})$ method was used.

Primer sequences to perform RT-qPCR were:

L1CAM: Fw-5'ACGAGGGATGGTGTCCACTTCAAA, Rev-5' TTATTGCTGGCAAAGCAGCGGTAG GAPDH: Fw-5' TCCCTGAGCTGAACGGGAAG, Rev-5'GGAGGAGTGGGTGTCGCTGT

\section{Antibody and immunohistochemistry}

The mouse monoclonal antibody anti-L1CAM, clone UMAB48, was purchased from OriGene Tchnologies
(Rockville, MD, USA). The formalin fixed paraffin-embedded (FFPE) tissue blocks were collected and cut into $5 \mu \mathrm{m}$ sections and mounted on Superfrost slides. Antigen retrieval was performed at $96{ }^{\circ} \mathrm{C}(10 \mathrm{mM} / \mathrm{L}$ citrate buffer, $\mathrm{pH}$ 6) for $20 \mathrm{~min}$. Sections were incubated with the primary antibody anti-L1CAM (1:30) for $30 \mathrm{~min}$ at room temperature. Bond Polymer Refine Detection Kit revealed immunoreaction according to manufacturer's procedure (Leica Biosystems) in an automated autostainer Bond III Leica Biosystems. Diaminobenzidine was used as chromogenic substrate. Microscope Nikon ECLIPSE 55i with digital camera HESP Technology was used. Scale bars $50 \mu \mathrm{m}$.

\section{MicroRNA analysis}

Reverse transcription and qRT-PCR amplification were performed in two steps. In the first reverse transcription step, 10 ng of RNA was used in reactions with specific stem-loop RT primer for miR-34a and endogenous control primer for small nuclear RNA U6. Reaction was performed with TaqMan MicroRNA Reverse Transcription Kit, according to the manufacturer's protocol (Applied Biosystems, Foster City, CA). In the second step, cDNA samples were amplified in Real Time PCR instrument 7500 (Applied Biosystems) with the specific TaqMan miR-34a assay and small nuclear RNA U6 as endogenous control previous tested to verify the minimal variation found among the tissues analyzed. The relative quantity (RQ) of each miRNA was calculated by the comparative CT (2- $\Delta \Delta \mathrm{CT})$ method.

\section{Statistical analysis}

Descriptive statistics were used to describe the patients' characteristics. Continuous variables are presented as median and range. Proportions are presented as numbers and percentages. Chi square test or Fisher's exact test, when appropriate, were used to estimate all association between categorical variables. Comparison between groups of continuous variables was performed by the use of Mann-Whitney U test. Receiver operating characteristic (ROC) analysis was performed to find the optimal cut-off for L1CAM expression and mRNA, capable of splitting patients into groups with different recurrence probabilities. Disease Free Survival (DFS) curves were estimated by the Kaplan-Meier product-limit, from the date of surgery until recurrence or last follow-up. The log-rank test was used to assess differences between subgroups. Significance was defined at the $p \leq 0.05$ level. A multivariate Cox proportional hazard model was developed using stepwise regression (forward selection). Variables testing significant at the univariate analysis were entered into the model, enter limit and remove limit were $P=0.10$ and $P=0.15$, respectively. The variables considered at univariate analysis included: age, tumor 
histology, grading, stage, Body Max Index (BMI), comorbidity (hypertension, diabetes), washing, lymph nodes, lymph vascular space invasion (LVSI), L1CAM expression. SPSS software (SPSS version 21.0, SPSS Inc., Chicago, Illinois, USA) and MedCalc ${ }^{\circ}$ (10.0.1) statistical programs was used for all analyses.

\section{Results}

A group of 113 EC patients (56 non-recurrent and 57 recurrent EC), median age 67 years (range 40-88) were included in this study and evaluated for DFS. The patients were stratified based on histology, grade of differentiation, FIGO stage, adjuvant therapy, lymph node positivity, washing, LVSI, hypertension, diabetes, and BMI (Table 1). The expression level of L1CAM protein was analyzed, by IHC analysis, in specimens derived from patients surgically treated at the "Regina Elena" National Cancer Institute between 2001 and 2014. Based on the ROC curve, optimal cut-off was determined at $20 \%$ for L1CAM (Additional file 1: Figure S1).

\section{L1CAM is higher expressed in recurrent compared to non-recurrent EC}

Among the recurrent EC samples analyzed, derived from FFPE tissues, we found 14 (24\%) of tumors with $\leq 20 \%$ of positive cells, $34(60 \%)$ with $>20 \%$ of positive cells, and 9 (16\%) negative tumors; among the non-recurrent EC analyzed samples we found and 19 (34\%) with $\leq 20 \%$ of positive cells, $13(23 \%)$ with $>20 \%$ of positive cells and $24(43 \%)$ negative tumors (Table 2). Figure 1a-d shows representative IHC analysis of L1CAM on recurrent (a-b) and non-recurrent (c-d) EC patient specimens, scored semi-quantitatively based on staining intensity $(0$, $1+, 2+$, and $3+$ ) and on the $\%$ of positivity.

\section{L1CAM expression correlates with lower DFS}

The median follow-up of the entire population was 48 months (range 1-162), the median DFS was 48 months (CI95\% 14-81). In the univariate analysis we found significant L1CAM expression, age, BMI, and LVSI and number of lymph node.

At multivariate analysis only L1CAM (HR 2.53, CI95\% $1.42-4.51, p=0.002)$ and age $>67$ years (HR 1.78, CI95\% $1.0-3.17, p=0.05)$ were confirmed as significant predictors of worse DFS (Table 3). When we correlated the expression of L1CAM in EC with DFS, we found that higher expression of L1CAM was present in tumor patients with lower disease free survival (5y-DFS $26.1 \%$ vs $60.7 \%, p=0.002$ ) (Fig. 2a). Furthermore, the correlation between age of patients and DFS highlighted those patients older than 67 years with worse survival (Fig. 2b). Even though we did not observe a significant correlation between L1CAM expression and age of patients $(p=$ 0.19 ), we observed a subgroup of patients with low
Table 1 Clinico-pathological characteristics of patients with endometrial cancer

\begin{tabular}{|c|c|c|}
\hline $\begin{array}{l}\text { Clinicopathological } \\
\text { characteristics }\end{array}$ & $\begin{array}{l}\text { No recurrence } \\
\mathrm{N}(\%)\end{array}$ & $\begin{array}{l}\text { Recurrence } \\
\text { N (\%) }\end{array}$ \\
\hline \multicolumn{3}{|l|}{ Age at diagnosis } \\
\hline median (range) & $65(40-84)$ & $68(48-88)$ \\
\hline$\leq 67$ yrs & $34(60.7)$ & $27(47.4)$ \\
\hline$>67 \mathrm{yrs}$ & $22(39.3)$ & $30(52.6)$ \\
\hline \multicolumn{3}{|l|}{ BMl } \\
\hline median (range) & $28(18-53)$ & $31(21-80)$ \\
\hline \multicolumn{3}{|l|}{ Grade } \\
\hline G1 & $1(1.8)$ & $6(10.6)$ \\
\hline G2 & 19 (33.9) & $17(29.8)$ \\
\hline G3 & $36(64.3)$ & $34(59.6)$ \\
\hline \multicolumn{3}{|l|}{ Histology } \\
\hline Adenocarcinoma (ADK) & $46(82.1)$ & $46(80.7)$ \\
\hline Others & $10(17.9)$ & $11(19.3)$ \\
\hline \multicolumn{3}{|l|}{ Stage } \\
\hline$\leq \mathrm{IB}$ & $21(37.5)$ & $32(56.1)$ \\
\hline$\geq \mathrm{IB}$ & $35(62.5)$ & $25(43.9)$ \\
\hline \multicolumn{3}{|l|}{ Adjuvant treatment } \\
\hline None & $11(19.6)$ & $15(26.3)$ \\
\hline Radiotherapy & 30 (53.6) & $22(38.6)$ \\
\hline Chemotherapy & $8(14.3)$ & $11(19.3)$ \\
\hline Radiation and chemotherapy & $7(12.5)$ & $9(15.8)$ \\
\hline \multicolumn{3}{|l|}{ Lymph nodes } \\
\hline Positive & $7(12.5)$ & $6(10.5)$ \\
\hline Negative & $49(87.5)$ & $51(89.5)$ \\
\hline \multicolumn{3}{|l|}{ Washing } \\
\hline Positive & $7(12.5)$ & $6(10.5)$ \\
\hline Negative & $49(87.5)$ & $51(89.5)$ \\
\hline \multicolumn{3}{|l|}{ LVSI } \\
\hline Positive & $13(23.2)$ & $13(22.8)$ \\
\hline Negative & $43(76.8)$ & $38(66.7)$ \\
\hline \multicolumn{3}{|l|}{ Hypertension } \\
\hline Yes & $27(48.2)$ & $33(57.9)$ \\
\hline No & $29(51.8)$ & $24(42.1)$ \\
\hline \multicolumn{3}{|l|}{ Diabetes } \\
\hline Yes & $6(10.7)$ & $9(15.8)$ \\
\hline No & $50(89.3)$ & $48(84.2)$ \\
\hline
\end{tabular}

L1CAM expression and age lower than 67 years at low risk of recurrence compared to a subgroup of patients with low L1CAM expression and age higher than 67 years with an increase of risk of recurrence (5-yrs DFS: 68.5 and 39.7\%, respectively) (Fig. 3a, b). When we stratified the expression of high L1CAM with the 
Table 2 L1CAM expression in recurrent and no recurrent EC

\begin{tabular}{|c|c|c|c|c|c|}
\hline & \multirow{2}{*}{$\begin{array}{l}\text { n (\%) } \\
\text { Negative }\end{array}$} & \multicolumn{2}{|l|}{ L1CAM expression n (\%) } & \multirow[t]{2}{*}{ n (\%) } & \multirow[b]{2}{*}{$P$ value } \\
\hline & & $\leq 20 \%$ of tumor cells & $>20 \%$ of tumor cells & & \\
\hline Recurrence & $9(16)$ & $14(24)$ & $34(60)$ & 57 & \\
\hline No recurrence & $24(43)$ & $19(34)$ & $13(23)$ & 56 & $<0.0001$ \\
\hline
\end{tabular}

grading of the disease, we found that higher L1CAM expression predicted a significant shorter DFS in the subgroup of patients with G3 tumor (5y-DFS, 67.7\% vs 16.6\%, $P=0.004$ ) (Fig. $3 \mathrm{~d}$ ); the expression of L1CAM did not predict risk of recurrence in $\mathrm{G} 1 / 2$ patients $(p=0.47)$ (Fig. 3c).

When we stratified the L1CAM expression according to the stage of the tumor $(<1 \mathrm{~B} v \mathrm{vs} \geq 1 \mathrm{~B})$ and the histotype
(ADK) we found that high level of L1CAM were associated with lower DFS in patients with stage of tumor $<1 \mathrm{~B}$ and G3 grading ( $p=0.006$ and $p=0.01$, respectively) (Fig. 4a and c); the expression of L1CAM did not predict risk of recurrence in patients with stage of the tumor $\geq 1 \mathrm{~B}$ or in not EC $(p=0.14$ and $p=0.25$, respectively $)$ (Fig. $4 \mathrm{~b}$ and d).

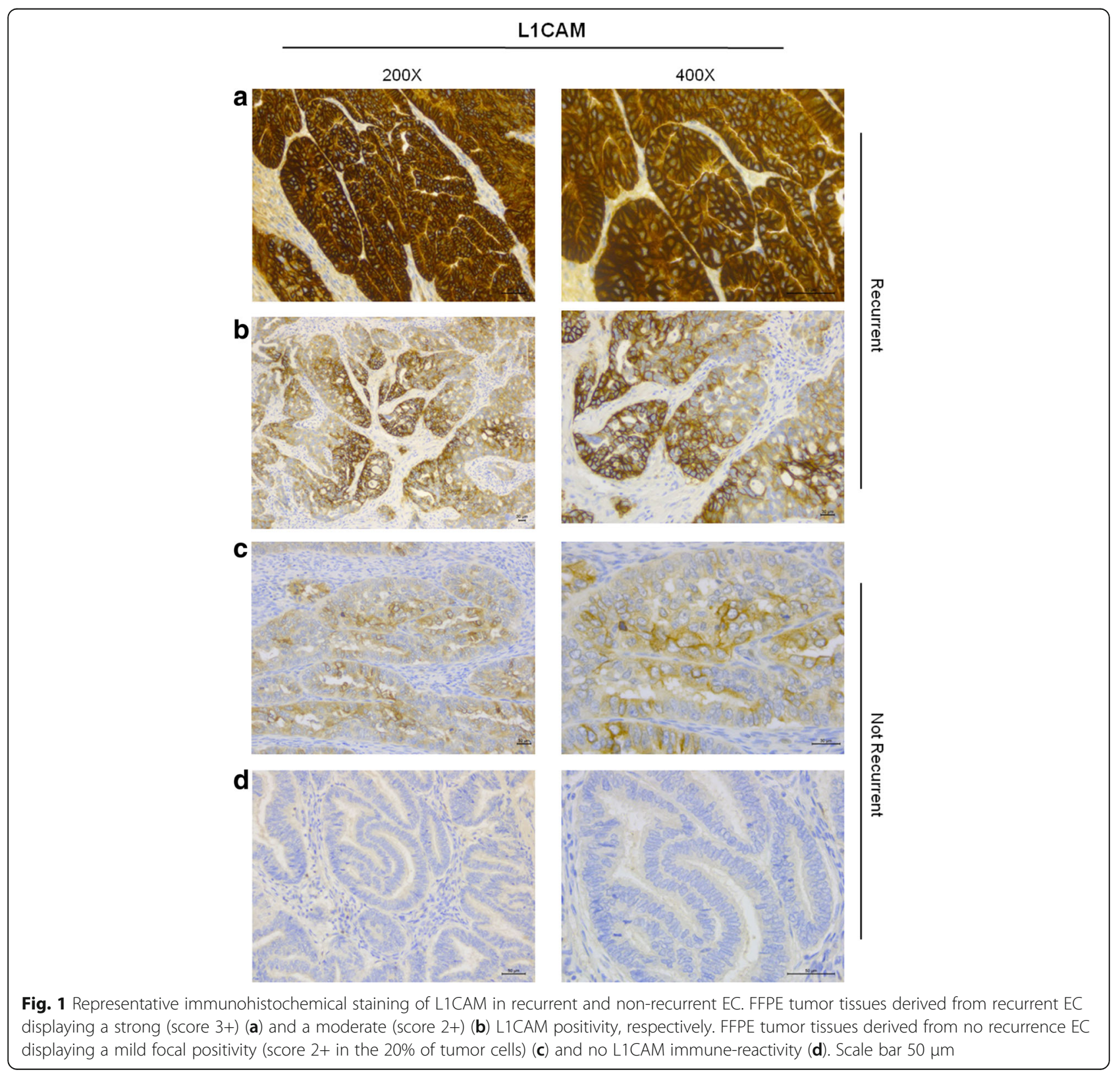


Table 3 Univariate and Multivariate analysis for Disease Free Survival

\begin{tabular}{|c|c|c|c|c|}
\hline \multirow[t]{3}{*}{ Variables } & \multicolumn{4}{|c|}{ Disease-free Survival } \\
\hline & \multicolumn{2}{|c|}{ Univariate analysis } & \multicolumn{2}{|c|}{ Multivariate Analysis } \\
\hline & HR (Cl95\%) & $P$ value & HR (Cl95\%) & $P$ value \\
\hline Age (>67 vs $\leq 67)$ & $2.32(1.32-4.07)$ & 0.003 & $1.78(1.0-3.17)$ & 0.05 \\
\hline Histotype (Other vs adenoc.) & $1.41(0.71-2.83)$ & 0.33 & - & NS \\
\hline Grading (3 vs 1-2) & $1.45(0.82-2.58)$ & 0.21 & - & NS \\
\hline Stage ( $>\mid B$ vs $\leq \mid B)$ & $1.48(0.81-2.69)$ & 0.20 & - & NS \\
\hline LICAM (> 20 vs $\leq 20)$ & $2.95(1.69-5.14)$ & $<0.0001$ & $2.53(1.42-4.51)$ & 0.002 \\
\hline BMI & $1.78(1.03-3.09)$ & 0.04 & - & NS \\
\hline Comorbidity (yes vs no) & $1.29(0.73-2.27)$ & 0.38 & - & NS \\
\hline Washing (yes vs no) & $1.12(0.58-2.19)$ & 0.73 & - & NS \\
\hline LVSI (yes vs no) & $2.0(1.12-3.53)$ & 0.02 & - & NS \\
\hline Number of lymph nodes & $0.98(0.95-0.99)$ & 0.04 & - & NS \\
\hline
\end{tabular}

High expression of miR-34a is a marker of good prognosis in EC

When we applied an explorative analysis, by RT-qPCR, we found that low level of L1CAM mRNA was expressed in recurrent compared to non-recurrent EC $(P=0.01)$ (Fig. 5). Based on the ROC curve, optimal cut-off for mRNA was determined at 0.893 (Additional file 2: Figure S2). The 5-years DFS was $64.3 \%$ for patients with high mRNA and 32\% for lower mRNA (Fig. 6a). Thus, we correlated the expression of L1CAM protein with the corresponding mRNA level and identified two subgroups of patients with low mRNA/highL1CAM (mRNA L/L1CAM $\mathrm{H}$ ) or high mRNA/lowL1CAM (mRNA H/L1CAM L) with different prognosis (Fig. 6b). The correlation of these data with DFS identified a subgroup of patients with mRNA L/L1CAM H protein that showed lower DFS $(P=$ 0.0003 ) suggesting a post-transcriptional regulation of L1CAM protein.

Thus, we analyzed the level of miR-34a in these subgroups of patients and found that miR-34a was down regulated in patients with mRNA L/L1CAM $\mathrm{H}$ and accumulated in patients with mRNA H/L1CAM L confirming that miR-34a controls L1CAM protein expression ( $p=0.01$, Fig. $6 c$ ). Surprisingly, we did not identify a subgroup of patients with mRNA L/L1CAM L (data not shown).

\section{Discussion}

Our study showed that L1CAM is highly expressed in vivo in recurrent compared to non-recurrent EC. We identified two groups of patients with different prognosis: high L1CAM expression and G3 with poor prognosis; low L1CAM expression and age lower than 67 years with good prognosis. Furthermore, we also found a subgroup of patients with low miR-34a/high L1CAM that showed lower DFS $(P=0.0003)$.

Although EC is characterized by a good prognosis, several authors, especially for early-stage EC, have reported a great heterogeneity of disease response [9]. EC site-specific recurrence patterns are influenced by classic prognostic factors such as histological type and grade, depth of myometrial invasion, LVSI, and nodal status $[10,11]$. It is now well
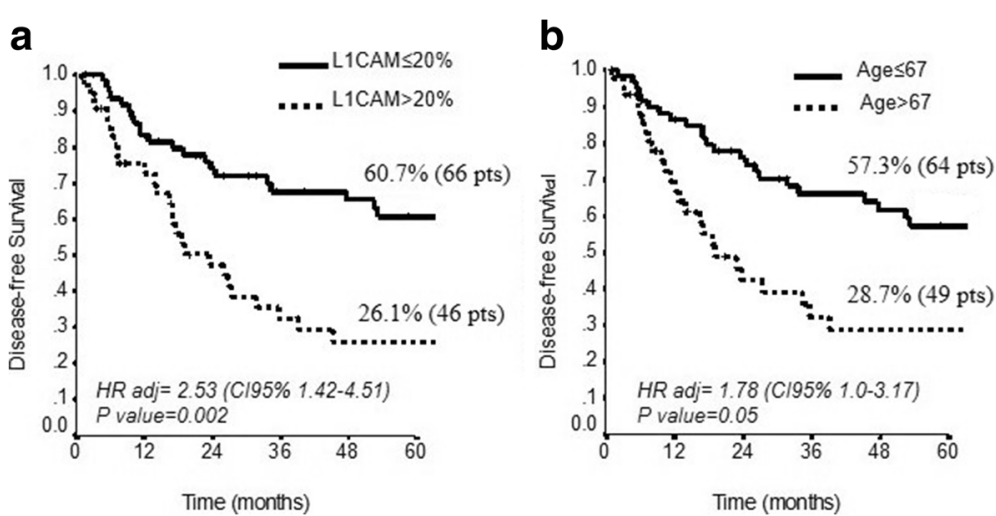

Fig. 2 The DFS according to L1CAM expression and age of patients. Kaplan-Meier estimate DFS for (a) L1CAM expression ( $\leq 20 \%$ vs $>20 \%$ of positive cells, respectively) $(P=0.002)$, and (b) age of the patients ( $\leq 67$ vs $>67)(P=0.05)$ 
L 1 CAM $\leq 20 \%$

a

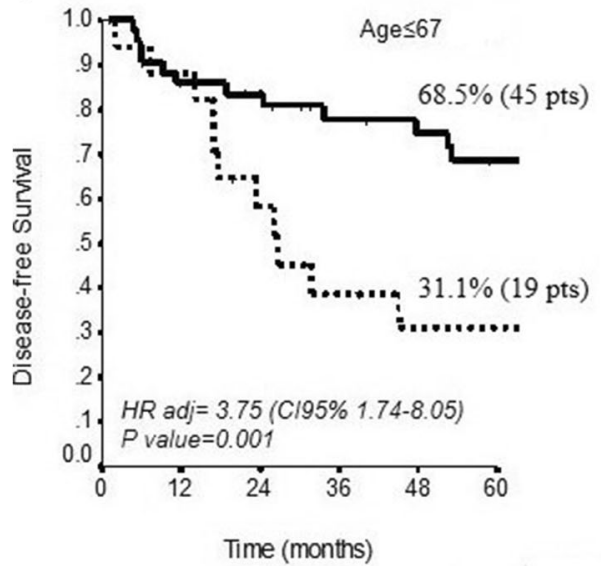

C

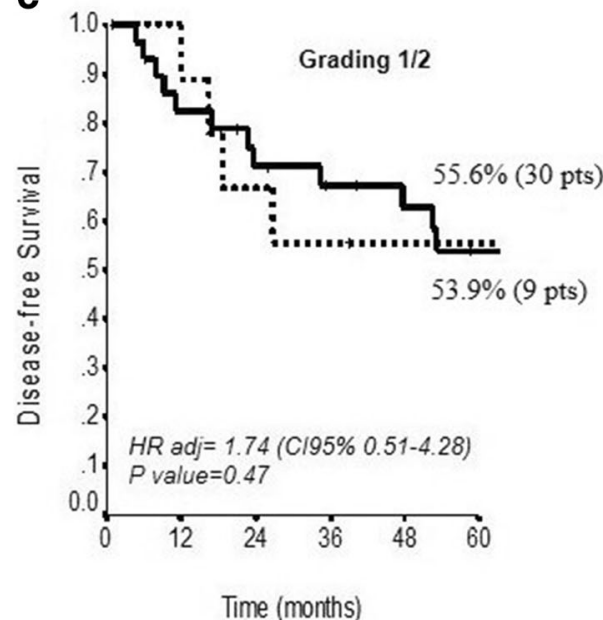

b

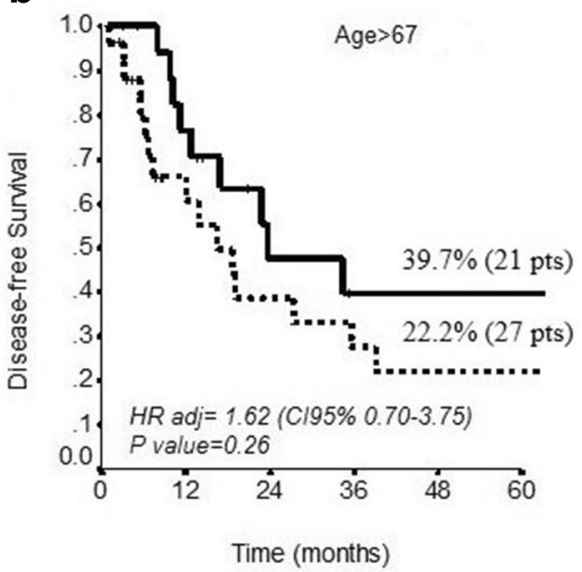

d

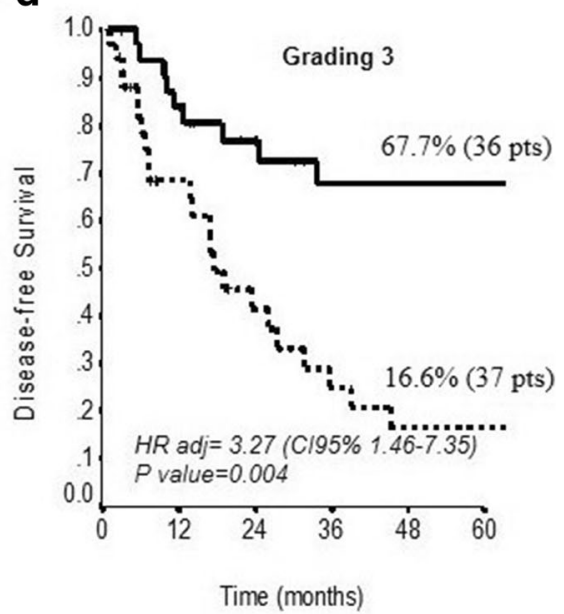

Fig. 3 The graph is representing DFS of L1CAM expression in patients of different age and tumor grade. $\mathbf{a}-\mathbf{b}$ Kaplan-Meier estimates DFS for L1CAM expression stratified for age of the patients (age $\leq 67$ years and age $>67$ years). c-d Kaplan-Meier estimate DFS for high vs low L1CAM expression stratified for grading (grading $1 / 2$ and grading 3). P-values were calculated using the log-rank test

established that recurrences after primary surgical treatment are mostly located in the true pelvis with events generally occurring in the regional pelvic lymph nodes or in the vaginal vault [12]. However, other locations including distant metastases or peritoneal carcinomatosis can also be observed underlining the prognostic heterogeneity of the disease [13]. In fact, several randomized trials [14-16] have shown no improvement of DFS both with adjuvant radiotherapy and with lymphadenectomy. Despite debatable selection criteria and methodological bias, it is likely that negative results observed in these studies are mainly related to the natural history of the disease that is characterized by a pattern of relapse more similar to ovarian cancer than to cervical cancer.

In the era of personalized approach and innovative biotechnology, expression of L1CAM has been associated with aggressive subtypes of endometrial carcinoma [5]. Moreover, L1CAM has shown to be of great importance for the prediction of clinical outcome in FIGO-stage I, histologically confirmed endometrioid endometrial cancer (EEC) [17]. L1CAM has been extensively investigated in the last 15 years in relation to its capacity in enhancing cell motility and thereby promoting invasiveness and its expression has been reported be associated with many cancers including breast, gastric and colorectal cancers [18, 19].

The IHC detection of L1CAM in endometrial tumour samples is able to discriminate a subset of highly aggressive tumours with adverse clinical outcome and high risk of distant recurrences, and to assess the risk of pelvic lymph-node involvement.

To assess whether the expression of L1CAM in vivo was higher in recurrent compared to not-recurrent EC, 


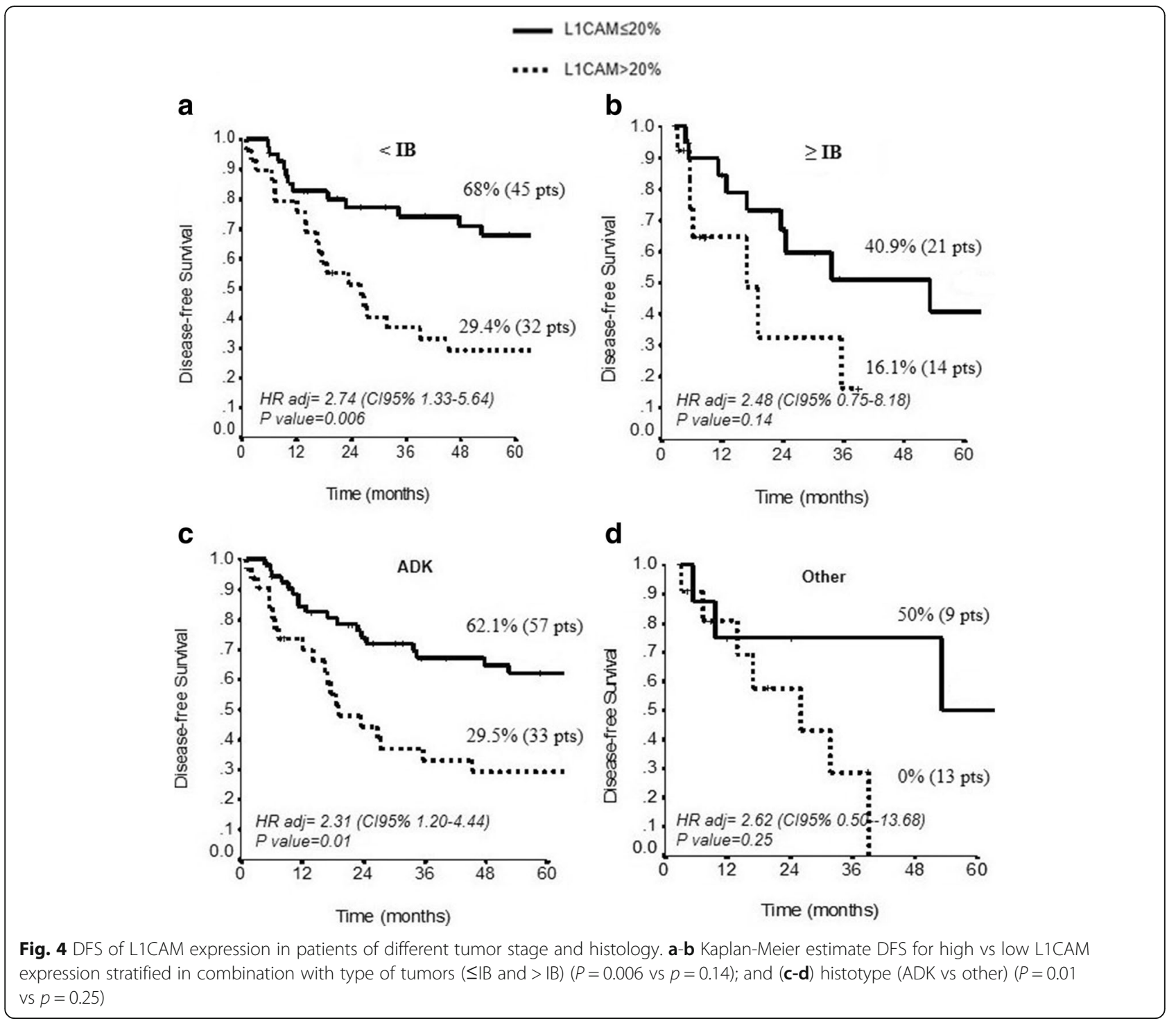

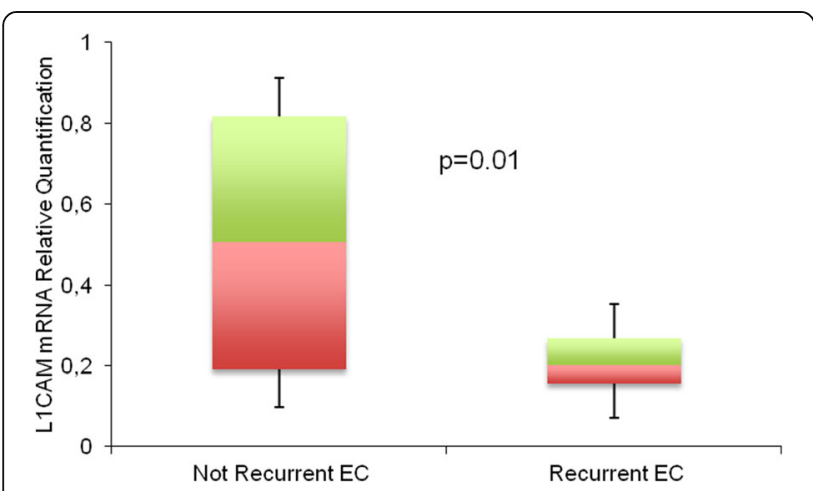

Fig. 5 L1CAM expression at the mRNA level on the cohort of 113 EC patients. Expression of L1CAM mRNA was examined by qRT-PCR on specimens derived from patients with no recurrence vs recurrent EC tumors $(P=0.01)$ we correlated the expression of the protein with the DFS. Our results confirm in a large single Institution series the prognostic role of L1CAM in EC patients. In our study, L1CAM seems to be highly predictive of tumour relapse in two groups of patients with opposite prognostic characteristics according traditional clinic-pathologic features:

- Patients $<67$ years old, usually considered at better prognosis compared with older patients

- Poorly differentiated tumours (G3) typically considered at bad prognosis.

Advanced age, in EC, has been associated with a number of poor pathologic features including aggressive tumour histology, increased tumour grade, and deep myometrial invasion [20]. In addition, elderly women are 


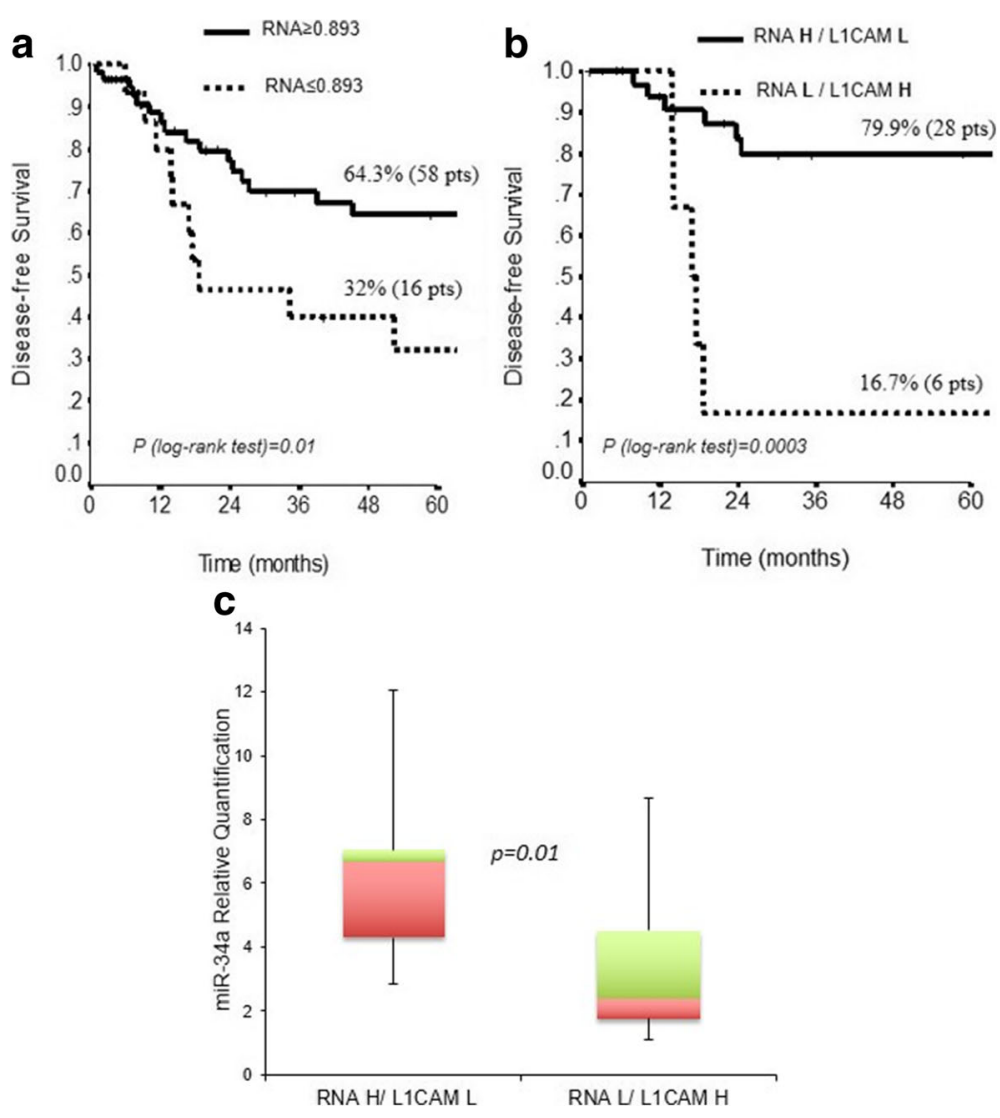

Fig. 6 DFS according to L1CAM mRNA and protein expression in EC specimens. The box plot represents the distribution of miR-34a in the two subgroups of patients. a Kaplan-Meier estimate DFS for the expression of low vs high L1CAM mRNA level $(P=0.01)$. b Kaplan-Meier estimate DFS for the expression of mRNA H/L1CAM L vs mRNA L/L1CAM H $(P=0.0003)$. $\mathbf{c}$ In the Box Plot are reported the miR-34a values in the two subgroups of patients (mRNA L/L1CAM H and mRNA H/L1CAM L) $(P=0.01)$

more likely to be diagnosed with advanced stage disease $[21,22]$. In our study EC low expressing L1CAM discriminate a group of patient with good prognosis when age lower than 67 years. High L1CAMs discriminate for a worse prognosis independently from age of patients.

High tumour grade and the presence of lymphovascular invasion remained as independent predictors of survival endpoints [23]. The association between L1CAM expression and higher grade has been shown by two recent reports including endometrial cancer of various histology and stages [24, 25]. Our study confirmed that L1CAM expression of the tumour is associated with poor differentiation and patients with L1CAM positive tumours were more likely to belong to groups of higher risk of relapse. Some authors suggest that grade 3 EEC may be more suitably considered non endometrioid endometrial cancer (NEEC) because they demonstrate similar immunohistochemical features and survival profile as those endometrial cancers traditionally considered more aggressive [26]. However, because the molecular profile of grade $3 \mathrm{EEC}$ has not yet been fully characterized, it does not clearly correspond to either definition of type I and II cancer [27]. A recent multicenter study of L1CAM-expression in 1021 histologically confirmed EEC demonstrated L1CAM positivity in $17.7 \%$ and demonstrated that L1CAM-expression in EEC was an independent predictor of clinical outcome. A small percentage of these cases showed areas of non endometrioid differentiation in less than $10 \%$ of the tumor, and this was associated with L1CAM-expression [28]. This and others studies suggested that L1CAM expression carries prognostic value for histologically classified EEC and supports the identification of tumors with a NEEC component [29].

L1CAM promotes cell motility, invasion, chemo-resistance and metastasis formation. Elucidating genetic processes involved in the expression of L1CAM in cancers is of considerable importance. There is also increasing evidence that micro-RNAs can also have strong effects on gene expression [30]. Remarkably, it was previously reported in primary tumor sections an inverse correlation between L1CAM protein and miR-34a expression [8]. Our results demonstrate that L1CAM per se is a prognostic factor of EC progression. However, the analysis of L1CAM mRNA and protein 
expression identified two subgroups of patients with poor (mRNA L/L1CAM H) and good (mRNA H/L1CAM L) prognosis. Furthermore, the analysis of miR-34a in these two subgroups of patients, revealed that miR-34a was expressed at low level in the mRNA L/L1CAM H subgroup corresponding to patients with lower DFS, suggesting a rapid translation of the mRNA that results in a strong increase of the protein expression. Furthermore, we cannot exclude that the low mRNA found in these tumors could be due to a rapid mRNA degradation. As expected, the miR3-4a was accumulated in mRNA H/ L1CAM L subgroup of EC patients with good prognosis; in these tumors the accumulation of the mRNA could be due to the high level of miR-34a that inhibits its translation maintaining high level of mRNA and low level of L1CAM protein. Our findings demonstrate that L1CAM and the miR-34a expression have a marked prognostic significance identifying specific subgroup of patients that might be directed with different therapeutic protocols.

\section{Conclusion}

The risk estimation in endometrial cancer is based on both preoperative and postoperative factors. In pre-operative setting L1CAM could be a useful additional tool. It could help to identify those EEC-patients who are at high risk of disease progression. Prospective studies may be needed to elucidate the value of L1CAM in predicting distant metastasis and to further investigate the effect of chemotherapy in patients with L1CAM positive tumors.

\section{Additional files}

Additional file 1: Figure S1. ROC curve identify the optimal cut off point for the expression of L1CAM (20\%). (TIF $99 \mathrm{~kb}$ )

Additional file 2: Figure S2. ROC curve identify the optimal cut off point for the expression of LICAM mRNA (0.893). (TIF $99 \mathrm{~kb}$ )

\section{Abbreviations}

DSF: Disease free survival; EC: Endometrial Cancer; EEC: Endometrioid endometrial cancer; FFPE: Formalin-fixed paraffin embedded; IHC: Immunohistochemical; L1CAM: L1-cell adhesion molecule; LVSI: Lymph vascular space invasion; NEEC: Non endometrioid endometrial cancer

\section{Availability of data and materials}

The datasets used and/or analyzed during the current study are available from the corresponding author on reasonable request.

\section{Authors' contributions}

GC, GC and RF conceived and designed the research project. RL, VL and MC performed the research. RF and GC wrote the manuscript. LDS, LC, and GP collected clinical sample. IS and AZ collected clinical data. GC, RF and EV revised the manuscript. All authors read and approved the final manuscript

\section{Ethics approval and consent to participate}

Experimental protocol was approved by the Ethics Committee of the Regina Elena National Cancer Institute (Rome, Italy), and performed in accordance with the relevant guidelines and regulations. Written informed consent was obtained from all patients.

Consent for publication

Not applicable.

\section{Competing interests}

The authors declare that they have no competing interests.

\section{Publisher's Note}

Springer Nature remains neutral with regard to jurisdictional claims in published maps and institutional affiliations.

\section{Author details}

'Department of Experimental Clinical Oncology, Gynecologic Oncology Unit, IRCCS - Regina Elena National Cancer Institute, Via Elio chianesi, 53 -, 00144 Rome, Italy. ${ }^{2}$ Department of Research, Advanced Diagnostics and Technological Innovation, Area of Translational Research, IRCCS - Regina Elena National Cancer Institute, Rome, Italy. ${ }^{3}$ Department of Research, Advanced Diagnostics and Technological Innovation, Anatomy Pathology Unit, IRCCS - Regina Elena National Cancer Institute, Rome, Italy. ${ }^{4}$ Scientific Direction, IRCCS - Regina Elena National Cancer Institute, Rome, Italy. ${ }^{5}$ Present address: Department of Women and Children Health, Gynecologic Oncology Unit, Fondazione Policlinico Universitario A. Gemelli - IRCCS, Università Cattolica del Sacro Cuore, Roma, Italy.

Received: 1 May 2018 Accepted: 28 June 2018

Published online: 06 July 2018

References

1. Siegel RL, Miller KD, Jemal A. Cancer statistics, 2016. CA Cancer J Clin. 2016; 66(1):7-30.

2. Bokhman JV. Two pathogenetic types of endometrial carcinoma. Gynecol Oncol. 1983;15:10-7.

3. Sherman ME. Theories of endometrial carcinogenesis: a multidisciplinary approach. Mod Pathol. 2000;13(3):295-308.

4. Cancer Genome Atlas Research N, Kandoth C, Schultz N, Cherniack AD, Akbani R, Liu Y, et al. Integrated genomic characterization of endometrial carcinoma. Nature. 2013:497(7447):67e73.

5. Huszar M, Pfeifer M, Schirmer U, Kiefel H, Konecny GE, Ben-Arie A, et al. Upregulation of L1CAM is linked to loss of hormone receptors and E-cadherin in aggressive subtypes of endometrial carcinomas. J Pathol. 2010;220(5): 551-61.

6. Bosse T, Nout RA, Stelloo E, Dreef E, Nijman HW, Jürgenliemk-Schulz IM, et al. L1 cell adhesion molecule is a strong predictor for distant recurrence and overall survival in early stage endometrial cancer: pooled PORTEC trial results. Eur J Cancer. 2014:50:2602-10.

7. Suh DH, Kim MA, Kim HS, Chung HH, Park NH, Song YS, et al. L1 cell adhesion molecule expression is associated with pelvic lymph node metastasis and advanced stage in diabetic patients with endometrial cancer: a matched case control study. J Cancer Prev. 2014;19:231-9.

8. Schirmer U, Doberstein K, Rupp AK, Bretz NP, Wuttig D, Kiefel H, et al. Role of MiR-34a as a suppressor of L1CAM in endometrial carcinoma. Oncotarget. 2014:5:462-7.

9. Colombo N, Creutzberg C, Amant F, Bosse T, González-Martín A, Ledermann $J$, et al. ESMO-ESGO-ESTRO consensus conference on endometrial cancer: diagnosis, treatment and follow-up. Ann Oncol. 2016;27(1):16-41.

10. Bendifallah S, Canlorbe G, Raimond E, Hudry D, Coutant C, Graesslin O, et al. A clue towards improving the European Society of Medical Oncology risk group classification in apparent early stage endometrial cancer? Impact of lymphovascular space invasion. Br J Cancer. 2014;110(11):2640-6.

11. Nugent EK, Bishop EA, Mathews CA, Einenkel J. Do uterine risk factors or lymph node metastasis more significantly affect recurrence in patients with endometrioid adenocarcinoma? Gynecol Oncol. 2012;125(1):94-8.

12. Creutzberg $\mathrm{CL}$, van Putten $\mathrm{WL}$, Koper $\mathrm{PC}$, Lybeert ML, Jobsen JJ, WárlámRodenhuis CC, et al. Surgery and postoperative radiotherapy versus surgery alone for patients with stage-1 endometrial carcinoma: multicentre randomised trial. PORTEC study group Post operative radiation therapy in endometrial carcinoma. Lancet. 2000;355(9213):1404-11.

13. Murali R, Soslow RA, Weigelt B. Classification of endometrial carcinoma: more than two types. Lancet Oncol. 2014;15(7):e268-78.

14. Benedetti Panici P, Basile S, Maneschi F, Alberto Lissoni A, Signorelli M, Scambia G, et al. Systematic pelvic lymphadenectomy vs. no lymphadenectomy in early-stage endometrial carcinoma: randomized clinical trial. J Natl Cancer Inst. 2008;100(23):1707-16.

15. ASTEC/EN.5 Study Group, Blake P, Swart AM, Orton J, Kitchener H, Whelan T, et al. adjuvant external beam radiotherapy in the treatment of endometrial 
cancer (MRC ASTEC and NCIC CTG EN.5 randomised trials): pooled trial results, systematic review, and meta-analysis. Lancet. 2009:373(9658):137-46.

16. Nout RA, Smit VT, Putter H, Jürgenliemk-Schulz IM, Jobsen JJ, Lutgens LC, et al. PORTEC study group. Vaginal brachytherapy versus pelvic external beam radiotherapy for patients with endometrial cancer of high-intermediate risk (PORTEC-2): an open-label, non-inferiority, randomised trial. Lancet. 2010 Mar 6;375(9717):816-23.

17. Smogeli E, Davidson B, Cvancarova M, Holth A, Katz B, Risberg B, et al. L1CAM as a prognostic marker in stage I endometrial cancer: a validation study. BMC Cancer. 2016;16:596.

18. Altevogt P, Doberstein K, Fogel M. L1CAM in human cancer. Int J Cancer. 2016;138(7):1565-76.

19. Wang YY, Li L, Zhao ZS, Wang YX, Ye ZY, Tao HQ. L1 and epithelial cell adhesion molecules associated with gastric cancer progression and prognosis in examination of specimens from 601 patients. J Exp Clin Cancer Res. 2013;32:66.

20. Bellino R, Arisio R, D'Addato F, Alba E, Attini R, Colla F, et al. Pathologic features of endometrial carcinoma in elderly women. Anticancer Res. 2001; 21:3721-4.

21. Mundt AJ, Waggoner S, Yamada D, Rotmensch J, Connell PP. Age as a prognostic factor for recurrence in patientswith endometrial carcinoma. Gynecol Oncol. 2000;79:79-85.

22. Benedetti Panici P, Basile S, Salerno MG, Di Donato V, Marchetti C, Perniola $\mathrm{G}$, et al. Secondary analyses from a randomized clinical trial: age as the key prognostic factor in endometrial carcinoma. Am J Obstet Gynecol. 2014; 210(4):363.e1-363.e10

23. Haley L, Burmeister C, Buekers T, Elshaikh MA. Is older age a real adverse prognostic factor in women with early-stage endometrial carcinoma? A matched analysis. Int J Gynecol Cancer. 2017;27(3):479-85.

24. Dellinger TH, Smith DD, Ouyang C, Warden CD, Williams JC, Han ES. L1CAM is an independent predictor of poor survival in endometrial cancer - an analysis of the Cancer genome atlas (TCGA). Gynecol Oncol. 2016;141(2):336-40.

25. Van Gool IC, Stelloo E, Nout RA, Nijman HW, Edmondson RJ, Church DN, et al. Prognostic significance of LICAM expression and its association with mutant p53 expression in high-risk endometrial cancer. Mod Pathol. 2016; 29:174-81.

26. Voss MA, Ganesan R, Ludeman L, McCarthy K, Gornall R, Schaller G, et al. Should grade 3 endometrioid endometrial carcinoma be considered a type 2 cancer-a clinical and pathological evaluation. Gynecol Oncol. 2012;124:15-20.

27. Felix AS, Weissfeld JL, Stone RA, Bowser R, Chivukula M, Edwards RP, et al. Factors associated with type I and type II endometrial cancer. Cancer Causes Control. 2010;21:1851-6.

28. Zeimet AG, Reimer D, Huszar M, Winterhoff B, Puistola U, Azim SA, et al. L1CAM in early stage type I endometrial cancer: results of a large multicenter evaluation. J Natl Cancer Inst. 2013;105(15):1142-50.

29. Geels YP, Pijnenborg JM, Gordon BB, Fogel M, Altevogt P, Masadah R, et al. L1CAM expression is related to non-Endometrioid histology, and prognostic for poor outcome in Endometrioid endometrial carcinoma. Pathol Oncol Res. 2016;22(4):863-8.

30. Sun X, Cui M, Zhang A, Tong L, Wang K, Li K, et al. MiR-548c impairs migration and invasion of endometrial and ovarian cancer cells via downregulation of twist. J Exp Clin Cancer Res. 2016;35:10.

\section{Ready to submit your research? Choose BMC and benefit from:}

- fast, convenient online submission

- thorough peer review by experienced researchers in your field

- rapid publication on acceptance

- support for research data, including large and complex data types

- gold Open Access which fosters wider collaboration and increased citations

- maximum visibility for your research: over $100 \mathrm{M}$ website views per year

At BMC, research is always in progress.

Learn more biomedcentral.com/submissions 